\title{
Building a numerical relativistic non-ideal magnetohydrodynamics code for astrophysical applications
}

\author{
S. Miranda Aranguren, M. A. Aloy and Carmen. Aloy \\ Departament d'Astronomia i Astrofisica Universitat de Valencia, \\ E-46100 Burjassot (Valencia) Spains \\ email: sergio.miranda@uv.es
}

\begin{abstract}
Including resistive effects in relativistic magnetized plasmas is a challenging task, that a number of authors have recently tackled employing different methods. From the numerical point of view, the difficulty in including non-ideal terms arises from the fact that, in the limit of very high plasma conductivity (i.e., close to the ideal MHD limit), the system of governing equations becomes stiff, and the standard explicit integrating methods produce instabilities that destroy the numerical solution. To deal with such a difficulty, we have extended the relativistic MHD code MR-GENESIS, to include a number of Implicit Explicit Runge-Kutta (IMEX-RK) numerical methods. To validate the implementation of the IMEX-RK schemes, two standard tests are presented in one and two spatial dimensions, covering different conductivity regimes.
\end{abstract}

Keywords. MHD, Relativity, numerical methods.

\section{Introduction}

The equations of Relativistic Magnetohydrodynamics form a system of balance laws where stiffness can arise because of the unbound increase of the electric conductivity, $\sigma$, in the ideal limit, which makes numerically ill-defined the electric density current, $\mathbb{J}=\sigma W[\mathbb{E}+\mathbb{V} \times \mathbb{B}-(\mathbb{E} \cdot \mathbb{V}) \mathbb{V}]+q \mathbb{V}$ (where $W, \mathbb{V}, q \mathbb{E}$, and $\mathbb{B}$ are the Lorentz factor, the velocity, the electric charge, the electric and the magnetic field, respectively). Inspired by the work of Dedner et al. (2002), we consider an augmented system of equations (see below), where two scalar potentials $\psi$ and $\phi$ enforce the conservation of $q$ and of the solenoidal constraint, $\nabla \cdot \mathbb{B}=0$, respectively, to the extent determined by the order of the numerical method employed. The inclusion of these extra potentials adds extra flux terms in the equations of $\mathbb{E}$ and $\mathbb{B}$, as well as additional source terms in the energy density $(\tau)$ equation, though the energy flux $\mathbb{F}_{\tau}$ remains unchanged. The rest of the equations for the rest-mass density $D$, and for the momentum density $\mathbb{S}$, and their respective fluxes $\mathbb{F}_{D}$, and $\mathbf{F}_{\mathbb{S}}$ are the same as in the standard equations.

To deal with the stiffness of the system, a number of alternatives have been proposed (Komissarov 2007, Palenzuela et al. 2009). We have included several Implicit-Explicit Runge Kutta methods (IMEX-RK; Higueras et al. 2012, Pareschi et al. 2005) in the MRGENESIS code (Aloy et al. 1999) and present several test problems.

$$
\begin{aligned}
& \partial_{t} \psi=-\nabla \cdot \mathbb{E}+q-\kappa \psi \\
& \partial_{t} \phi=-\nabla \cdot \mathbb{B}-\kappa \phi \\
& \partial_{t} \mathbb{E}=\nabla \times \mathbb{B}-\nabla \psi-\mathbb{J} \\
& \partial_{t} \mathbb{B}=-\nabla \times \mathbb{E}-\nabla \phi
\end{aligned}
$$

$\partial_{t} q=-\nabla \cdot \mathbb{J}$

$\partial_{t} D=-\nabla \cdot \mathbb{F}_{D}$

$\partial_{t} \tau=-\nabla \cdot \mathbb{F}_{\tau}-\mathbb{B} \cdot \nabla \phi-\mathbb{E} \cdot \nabla \psi$

$\partial_{t} \mathbb{S}=-\nabla \cdot \mathbf{F}_{\mathbb{S}}-(\nabla \cdot \mathbb{B}) \mathbb{B}$ 

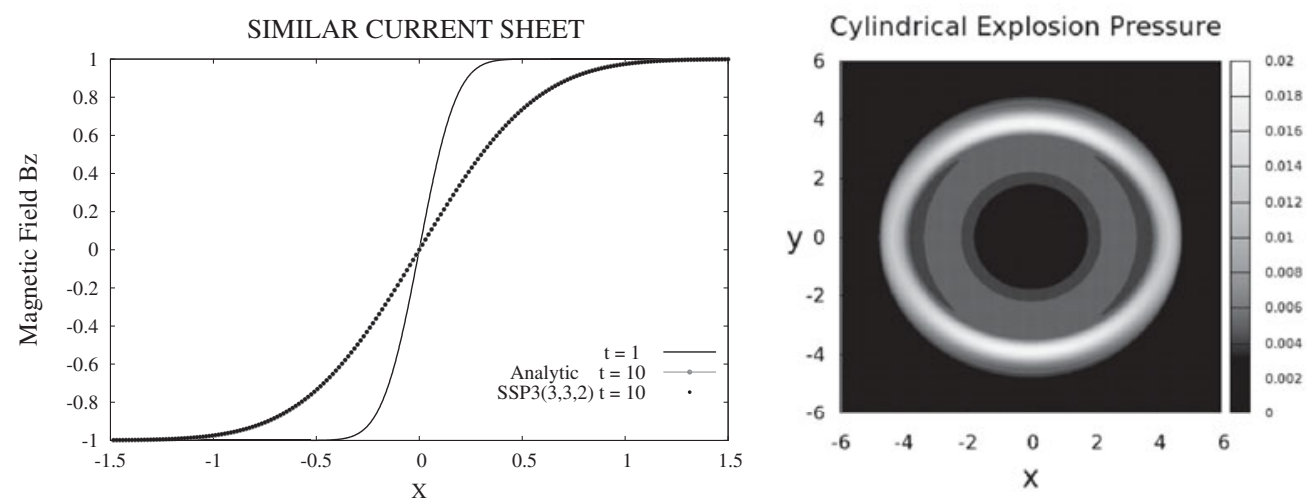

Figure 1. Left: Self-similar current sheet at $t=1$ (initial state; solid line), analytic solution at $t=10$ (open circles) and numerical solution computed with 800 points at $t=10$ (filled circles). Right: Thermal pressure for the cylindrical explosion test computed with a uniform grid of $400 \times 400$ cells, and a CFL of 0.2 , and using the DP2A(242) scheme.

\section{Test Problems}

Due to space restrictions, we only show a couple of standard tests also considered by other authors (Komissarov 2007, Palenzuela et al. 2009, Bucciantini \& Del Zanna 2013). These tests encompass from one-dimensional, smooth flows driven by resistive effects (Sect. 2.1) to two-dimensional tests in the ideal limit (Sect. 2.2).

\subsection{Resistive Self-similar Current Sheet}

The initial conditions of this test are set at $t=1$, when we take $\sigma=100, \rho=1, p=50$, $\mathbb{E}=\mathbb{V}=0$ and $\mathbb{B}=\left(0,0, B^{z}(x, 1)\right)$, such that $B^{z}(x, t)=B_{0} \operatorname{erf}\left(\frac{1}{2} \sqrt{\frac{\sigma x^{2}}{t}}\right)$, where erf is the error function. In Fig. 1 (left) we show that at $t=10$, the numerical solution found with the SSP3(3,3,2) IMEX-RK method matches satisfactory the analytical one.

\subsection{Cylindrical Explosion}

The set up consists of a plane with dimensions $(x, y) \in[-6,6]$ having a central circle with radius $r=0.8$, where the pressure $(p=1)$ and the density $\rho=0.01$ are higher than elsewhere $(p=\rho=0.001 ; r>1)$. The central region is continuously connected with the surroundings using an exponentially decreasing pressure and density in the region $0.8 \leqslant r \leqslant 1$. The magnetic field, $\mathbb{B}=(0.05,0,0)$, is uniform, and $\mathbb{V}=\overrightarrow{0}$. This test is used to validate the new resistive code in the ideal limit (a uniform conductivity $\sigma=10^{6}$ is set everywhere). Our results (Fig. 1 right) are comparable with the those obtained with our relativistic ideal MHD code (Leismann et al. 2005, Anton et al. 2010).

\section{References}

Aloy, M. A., et al. 1999, ApJS, 122,151

Anton, L., et al. 2010, ApJS, 188, 1

Bucciantini, N., Del Zanna L. 2013, MNRAS, 428, 71

Dedner, A., et al. 2002, J. Comput. Phys., 175, 645

Higueras, I., et al. 2012, ASC Report No. 14/2012

Komissarov, S. S. 2007, MNRAS, 382, 995

Leismann, T. et al. 2005, Astron. Astroph., 436503

Palenzuela, C., et al. 2009, MNRAS, 394, 1727

Pareschi, L., et al. 2005, J. Sci. Comput., 25, 112 